\title{
Mental Health Assistance
}

\author{
Dr.Simran Khaini ${ }^{1}$, Kaushal Bhagvatprasad Chindak ${ }^{2}$, Atharva Shivaji Jadhav ${ }^{3}$, \\ Sushrut Avinash Borle ${ }^{4}$, Varad Shivaji Shenkude ${ }^{5}$ \\ ${ }^{1,2,3,4,5}$ G.H Raisoni College Of Engineering And Management, Wagholi , Pune
}

\begin{abstract}
We propose a system for a virtual mental health assistant Diagnose owing to time and space constraints and shortage of resources related to in-person therapy.

Oftenly, disturb mental health is a snowball effect built up over time and requires continuous attention and conscious efforts to improve. This is possible with the help of a virtual mental health Diagnosis.

For detection and classification of various mental health problems, machine learning algorithms are utilized via training of various models which work in question-answer manner.

The purpose of Diagnostic will have a Test regarding your problems which can determine the level of problems and accordingly provide solutions, psychological assessment, an emotion detection module and a recommendation system for improving the mood of the user. User will have to answer some particular questions related to mental health.
\end{abstract}

Keywords: Mental health, Virtual mental health assistant, Virtual Diagnosis, Classification, Machine Learning

\section{INTRODUCTION}

The COVID-19 pandemic has had a major effect on our lives. Many of us are facing challenges that can be stressful, overwhelming, and cause strong emotions in adults and children.

Public health actions, such as social distancing, are necessary to reduce the spread of COVID-19, but they can make us feel isolated and lonely and can increase stress and anxiety.

This System also intend to help you to identify your problems and get piece of advice in addition with interaction with community.

Machine Learning in Mental Health

1 ) Machine learning advances are opening new routes to more precise mental healthcare, from the discovery of disease subtypes to the development of personalised interactions supporting self-care between clinic visits. This offers an exciting opportunity for machine learning techniques to impact healthcare in a meaningful way.

2) High prevalence of mental illness and the need for effective mental health care, combined with recent advances in AI, has led to an increase in explorations of how the field of machine learning (ML) can assist in the detection, diagnosis and treatment of mental health problems.

4) Overall, the application of ML to mental health has demonstrated a range of benefits across the areas of diagnosis, treatment and support, research, and clinical administration. With the majority of studies identified focusing on the detection and diagnosis of mental health conditions.

\section{LITERATURESURVEY:}

\begin{tabular}{|l|l|l|l|}
\hline Sr no & $\begin{array}{l}\text { Published } \\
\text { Year } \\
\text { [Referance } \\
\text { s] }\end{array}$ & Methodology & Description \\
\hline 1 & $\begin{array}{l}\text { (2019) }[1] \\
\text { Gradient Boosting Machine } \\
(\mathrm{GBM})(2019)\end{array}$ & $\begin{array}{l}\text { GBM algorithm is frequently used in field of } \\
\text { mental health. Gradient boosting machines are a } \\
\text { family of powerful machine-learning techniques } \\
\text { that have shown considerable success in a wide } \\
\text { range of practical applications. They are highly } \\
\text { customizable to the particular needs of the } \\
\text { application, like being learned with respect to } \\
\text { different loss functions. }\end{array}$ \\
\hline
\end{tabular}


Vol. 10, Issue 11, November 2021

DOI: 10.17148/IJARCCE.2021.101118

\begin{tabular}{|c|c|c|c|}
\hline 2 & (2016) [2] & $\begin{array}{l}\text { A Random Forest Model for } \\
\text { Mental Disorders Diagnostic } \\
\text { Systems (2016) }\end{array}$ & $\begin{array}{l}\text { Random Forest is an ensemble machine learning } \\
\text { method developed by Leo Breiman in } 2001 \text {. } \\
\text { Since then, it has been considered the state-of-the- } \\
\text { art solution in machine learning applications. } \\
\text { Compared to the other ensemble methods, random } \\
\text { forests exhibit superior predictive } \\
\text { performance. }\end{array}$ \\
\hline 3 & (2013) [3] & $\begin{array}{l}\text { Mental Health Assessment Using } \\
\text { Support Vector Machines }\end{array}$ & $\begin{array}{l}\text { Evaluate the SVM classifiers as to their ability to } \\
\text { predict SLD items on two mental health } \\
\text { assessments: the Thought, Language and } \\
\text { Communication Scale (TLC) and the Clinical } \\
\text { Language Disorder Rating Scale (CLANG). }\end{array}$ \\
\hline 4 & (2019) [4] & $\begin{array}{l}\text { Emotion Recognition } \quad \text { Naïve } \\
\text { Bayes' Classifier }\end{array}$ & $\begin{array}{l}\text { Naïve bayes classifierto identify state of mental } \\
\text { health in a target group. The responses obtained } \\
\text { from the target group for the designed } \\
\text { questionnaire were first subject to unsupervised } \\
\text { learning techniques. The labels obtained as a } \\
\text { result of clustering were validated by computing } \\
\text { the Mean Opinion Score. }\end{array}$ \\
\hline 5 & (2018) [5] & $\begin{array}{l}\text { Detecting Depression Using K- } \\
\text { Nearest Neighbors } \quad \text { (KNN) } \\
\text { Classification Technique } \\
\text { (2018) }\end{array}$ & $\begin{array}{l}\text { NCapture to collect data and KNN approach } \\
\text { for extracting paraphrases to detect emotions } \\
\text { from user comments. Basically, we focused on } \\
\text { three basic emotions based on emotional } \\
\text { process, temporal process, and linguistic } \\
\text { analysis }\end{array}$ \\
\hline
\end{tabular}

\section{- GOALS \&OBJECTIVES}

We aim to develop an system that diagnose mental health related issues, accordingly provide solutions, psychological assessments, emotion detection module and recommendation module to help cure mental health problems.

\section{- SCOPE}

- Users can interact with anyone or put their thoughts on particular groups determined on mental stress or addiction anonymously if they wish.

- This system can be used in organizations in order to take weekly assessments and to maintain proper mental health

- This system have numerous usability scenarios and can be used in most of the fields as it intend to provide user friendly and interactive user interface.

\section{- MOTIVATION}

Just as the human body has physical biomarkers, it has behavioral biomarkers to conditions such as depression and anxiety. Machine learning algorithms could help determine key behavioral biomarkers to aid mental health professionals in deciding if a patient is at risk of developing a particular mental health disorder.

\section{- EXISTINGSYSTEM}

Existing virtual mental health systems mainly focuses on detection and compromises with user interaction and vast range of effective solutions. Also it rarely provides modules to share their mental health related problems with community.

Existing systems for virtual mental health fails to provide accurate test result due to insufficient datasets ( 89.20 $91.50 \%$ )

\section{- DISADVANTAGES}

1. System does not provide any prescribed medication by itself to help improve mental health.

However we will also get certified Doctors who are willing

to give free advice to the people who are going through mental issues or Addiction.

\section{- PROPOSEDSYSTEM}

Supervised machine learning, used for classification or prediction modelling, has the advantage of accounting for complex relationships between variables that may not have been previously identified. Thus, as datasets become larger and the variables more complex, machine learning techniques may become a useful tool within psychiatry to properly disentangle variables associated with outcomes for patients.

- Datasets :- The two main phases are- 1) To make a dataset of questions and train the model. 2) match the results of test with the dataset, calculating the accuracy and then giving result to the user. Prediction model could be 
Vol. 10, Issue 11, November 2021

DOI: $10.17148 /$ IJARCCE.2021.101118

based on several machine learning techniques like Random Forest and SVM to determine which produced the best fitting model for a test set. The model with the lowest error was then tested using the tune set. Once the performance in the tune set was deemed satisfactory, the final models were then fitted to the test set.

- $\quad$ Preparation of Training dataset:- Splitting our data into training set allows more accurately predict other databases. Most machine learning techniques require complete datasets, missing values will be imputed. Loading dataset in proposed system and creating predictions test cases

- ADVANTAGES

1) More accuracy than existing models.

2) It provides recommendation module for respective emotional states.

- SYSTEMARCHITECTURE

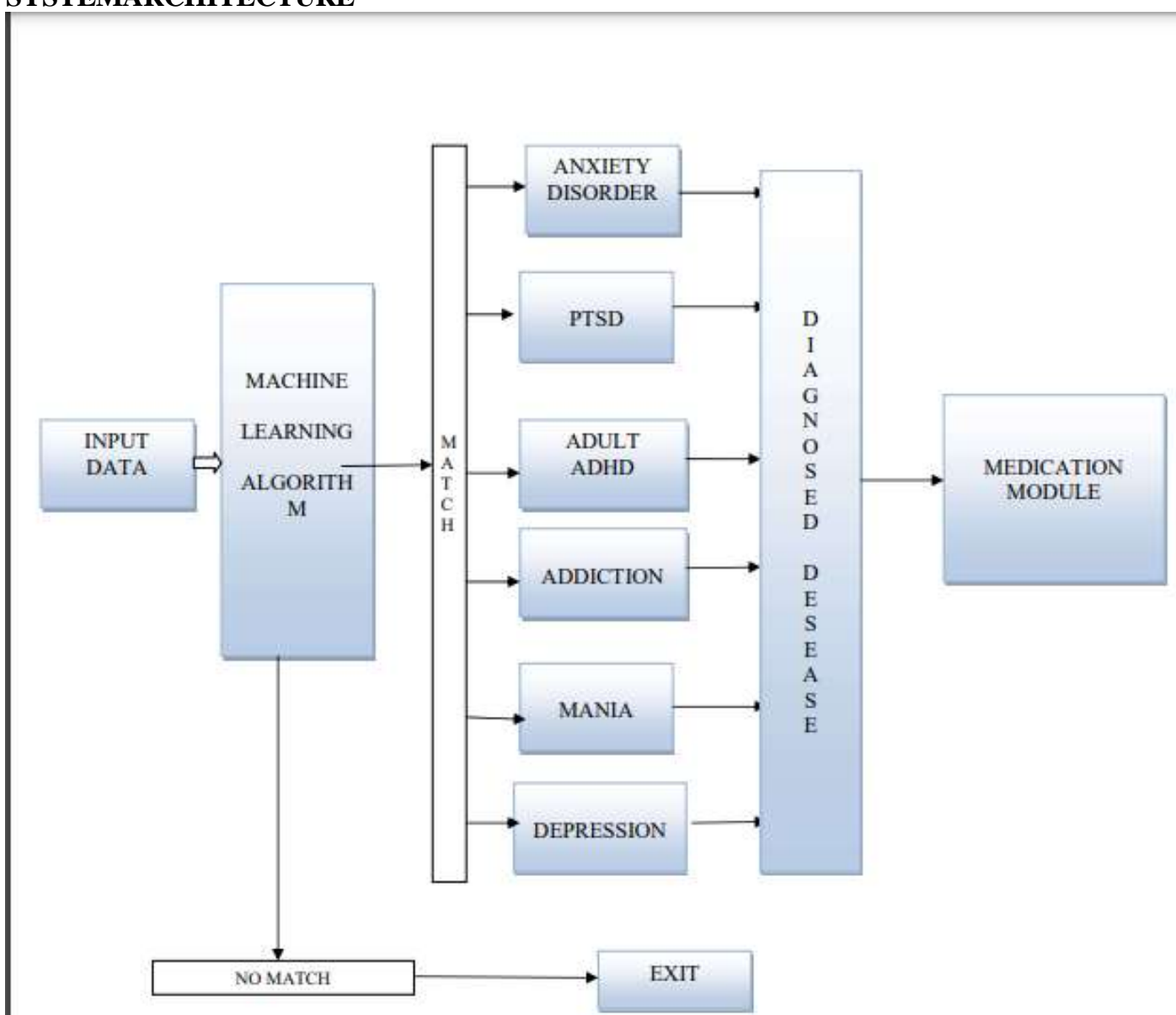

\section{- SYSTEMREQUIREMENTS}

\section{Hardware Requirements}

$\begin{array}{lll}\text { 1. } & \text { SystemProcessors } & \text { Intel i5 } 5^{\text {th }} \text { Gen } \\ \text { 2. } & \text { Speed : } & 3.2 \mathrm{GHz} \\ \text { 3. } & \text { HardDisk } & 500 \mathrm{~GB}\end{array}$




\section{International Journal of Advanced Research in Computer and Communication Engineering}

Vol. 10, Issue 11, November 2021

DOI: $10.17148 /$ IJARCCE.2021.101118

\section{Software Requirements}

1. Operatingsystem : 64 bit Windows 10

2. Coding Language : Python

3. Designconstraint : Anaconda,TensorFlow

\section{ACKNOWLEDGMENT}

We here by wish to take this opportunity to express our gratitude to our teachers and friends and all who have helped toward the completion of our project.

We take a great honor in presenting this Project Report to our Director, Dr. Vaibhav Hendre.

We also like to give thanks to our Guide Ms. Simran Khiani for helping and guiding us throughout our endeavor.

We are very grateful to our teaching staff for guiding us all over the duration of the degree. They were very helpful to us, as and when we required their help. We are also very grateful to non-teaching staff to help us in the laboratory in various ways.

We would also like to extend our gratitude to those friends whose knowledge and time helped us in many different ways.

\section{CONCLUSION}

Development of a Virtual Mental Health Assistant. It focus on improving the drawbacks of existing Mental health using machine learning systems accordingly provide solutions, psychological assessments, emotion detection module and recommendation module to help cure mental health problems.

\section{REFERENCES}

[1] Vidit Laijawala, Aadesh Aachaliya, H. A. Jatta, "Classification Algorithms based Mental Health Prediction using Machine Learning," 2020 5th International Conference on Communication and Electronics Systems (ICCES),2020.

[2] Ashley E. Tate, Ryan C. McCabe, Henrik Larsson, Ralf Kuja-Halkola "Predicting mental health problems in adolescence using machine learning" Journal- journal.pone. 02303892020

[3] M. Srividya, S. Mohanavalli, N. Bhalaji, "Behavioral Modeling for Mental Health using Machine Learning Algorithms" Journal of Medical Systems, IEEE2018

[4] Anja Thieme, Danielle Belgrave,Gavin Doherty "Machine Learning in Mental Health: A Systematic Review of the HCI Literature to Support the Development of Effective and Implementable ML Systems" ACM Transactions on Computer-Human InteractionVolume 27Issue Article No.: 34 pp $1-53,2020$

[5] Gyeongcheol Cho, Jinyeong Yim, Younyoung Choi, Jungmin Ko, and Seoung-Hwan Lee," Review of Machine Learning Algorithms for Diagnosing Mental Illness" 2019. 